\title{
Performance Measures of the Tomographic Classifier Fusion Methodology
}

\author{
DAVID WINDRIDGE ${ }^{1}$ \\ Department of Electronic Engineering, \\ University of Surrey, Guildford, \\ Surrey, GU2 7XH, \\ United Kingdom. \\ D.Windridge@surrey.ac.uk \\ JOSEF KITTLER ${ }^{2}$ \\ Department of Electronic Engineering, \\ University of Surrey, Guildford, \\ Surrey, GU2 7XH, \\ United Kingdom. \\ J.Kittler@surrey.ac.uk
}

\begin{abstract}
We seek to quantify both the classification performance and estimation error robustness of the authors' tomographic classifier fusion methodology by contrasting it in field tests and model scenarios with the sum and product classifier fusion methodologies.

In particular, we seek to confirm that the tomographic methodology represents a generally optimal strategy across the entire range of problem dimensionalities, and at a sufficient margin to justify the general advocation of its use. Final results indicate, in particular, a near 25\% improvement on the next nearest performing combination scheme at the extremity of the tested dimensional range.
\end{abstract}

Keywords: multiple classifier systems; optimization; performance assessment; tomography, estimationerror assessment

\section{Introduction}

We have, in a series of previous papers [1-6], described a mathematical analogy between, respectively, the processes of feature selection and Bayesian classifier combination, and the processes of Radon transformation and back-projection familiar from medical imaging. The entity which is thus implicitly reconstructed by back-projection/classifier-combination is the prior probability density function pertaining to the entirety of the pattern-space before feature-selection took place (giving rise to a series of predominantly disjoint feature sub-sets represented within individual classifiers: see [7-10] for evidence of the likelihood of this configuration when feature-selection explicitly considers the combined classifier output). The individual classifiers are thus, in this sense, 'morphological information probes' of the underlying composite pattern space PDF, since we expect feature selection to occur on the basis of the representative ability of the classifiers. The papers [1-6] thus sought to optimize the process of classifier combination on purely morphological grounds by introducing a filtration process to eliminate arbitrary morphology imposed by the mechanics of specifically linear back-projection upon on the recovered composite PDF (all extant combination schemes having been demonstrated to exhibit this linearity of back-projection). The chief motivating factor behind this approach is thus to derive and optimize the

\footnotetext{
${ }^{1}$ Present address.

${ }^{2}$ Permanent address.
} 
classifier-combination process on a priori grounds, rather than seeking optimization from within (cf eg 11-15]) a preexisting combination strategy selected on contingent or heuristic grounds.

In the case of the sum rule combination scheme, the mathematical analogy with back-projection is exact (the remaining combination schemes (eg [16-21]) having been demonstrated to act as specifically constrained forms of back-projection), and the proposed 'tomographically-filtered' combination scheme thus acts in relation to this particular fusion methodology. Precise algorithmic details of the methodology arising from this approach are set out in [4], the main results of which are reprinted (with permission) in appendix 1, which will thus serve in the current scenario as the test-bed tomographic combination methodology through which we shall address the paper's objectives. (It should be noted in passing that the appendix's technique is highly optimized, and superficially betrays little of its tomographic pedigree: interested readers are thus referred to [4] for precise details of its derivation and relation to tomography. Cursory inspection of the economized procedure should, however, reveal that it seeks, like tomography, to correlate related morphology across all of the classifiers' feature spaces in a manner not feasible for any simply linear combination).

\subsection{Endeavor of the Current Paper}

It was stated without proof in the papers [1-6] that the expected error resilience of the tomographic method ought to be similar to that of the sum rule, the optimal combination strategy in terms of robustness to estimation error [1], since the back-projection aspect of the tomographic fusion approach imposes exactly the same averaging process with respect to stochastic variation. A precise calculation was omitted since it depends critically on the interaction between the filtering mechanism and the morphological characteristics of the classifier (which is not something we would wish to specify in advance, the tomographic method being intended as a 'black box' approach, to which novel methods of classification may be appended as developed). Given this theoretical limitation on characterizing the error resilience of the proposed method, it is necessary to base any attempted quantification of the resilience to estimation error instead on practical trials and model solutions in order to build a convincing case.

More generally, though, we have yet to fully establish the most significant performance statistic for the tomographic combination method in relation to the conventional alternatives: the effect on the misclassification rate. A very limited example of such statistics within an applications context were given in [1] for the two-dimensional case (that is, the combination of two classifiers containing a single feature). However, an inspection of the methodology set out in the appendix would indicate that it is not possible to guarantee an equivalent performance response for combinations of higher dimensionality without a great deal of further analysis. Indeed, this is self-evidently not the case if the classifiers constituting the combination exhibit any degree of estimation error, since error resilience scales differently with dimensionality for the sum and product rule combination schemes (see discussion in [22]). It is therefore necessary, in any reasonable attempt to quantify the general performance of tomographic combination, to establish performance across the range of feature-space dimensionalities: we should in particular like an assurance that the tomographic method remains the optimal choice at higher dimensionalities within a representative range of scenarios. Sections 2 and 3 of the current paper shall therefore, respectively, detail our attempts to achieve this at the practical and theoretical levels.

Giving any comparative performance benchmark for the tomographic combination method requires that we test it against a representative sample of the remaining combination schemes. Kittler et al. [17] have demonstrated that the majority of commonly used decision rules can be derived mathematically from either the sum or the product decision rules, or else from their combination. We may therefore argue that these two methods collectively represent the full gamut of combinatorial methodologies, and that throughout the following paper it should therefore be these two characteristic methodologies against which we benchmark the tomographic combination system of appendix 1 . In doing so it will be 
seen that the product rule decision scheme may be reinterpreted within the terms of our inquiry as the attempt to reconstruct the higher-dimensional PDF of the composite feature-space (on the assumption of feature independence), in contrast to which the sum rule decision scheme can be understood as exemplifying the objective of estimation error cancellation (as is perhaps most intuitively evident with respect to high-variance classifiers occupying the same feature-space). Both of these means to increasing the classification performance of composite classifiers are distinct and complementary; we shall hence wish, throughout the following, to demonstrate that the tomographic methodology embodies both of these principles to the fullest extent.

\section{Investigation of Relative Real-world Performance of Tomo- graphic Classifier Fusion}

\section{$2.1 \quad$ Test Data}

For the practical, as opposed to the mathematical aspect of this investigation, the 'real-world' data upon which we shall perform this experimental comparison derives from a set of expertly-classified geological survey images, with the 26 dimensions of the pattern vectors corresponding to 26 distinct cell-based processes for texture characterization. The arbitrary nature of these processes means that the data simultaneously exhibits all three of the distinct characteristics of, respectively; large-scale feature redundancy, (relative) feature independence and its converse, feature dependency within its various feature subsets: that is, very largely the full range of possible behaviors with regard to feature selection, classification and classifier combination. An indication of the morphological complexity of the data in relation to two arbitrarily-selected features can be gained by inspection of Figure 1. It is evident even from this limited sample that the assumption inherent in the product rule's implicit attempt at recomposition of the overall feature-space's probability density function, namely the statistical independence of the marginal classifiers, can at best only approximately true for the data as a whole. We argue that, in this respect at least, the feature dependence of the chosen data-set is broadly representative of the generality of data-sets typically brought before the pattern-recognition community.

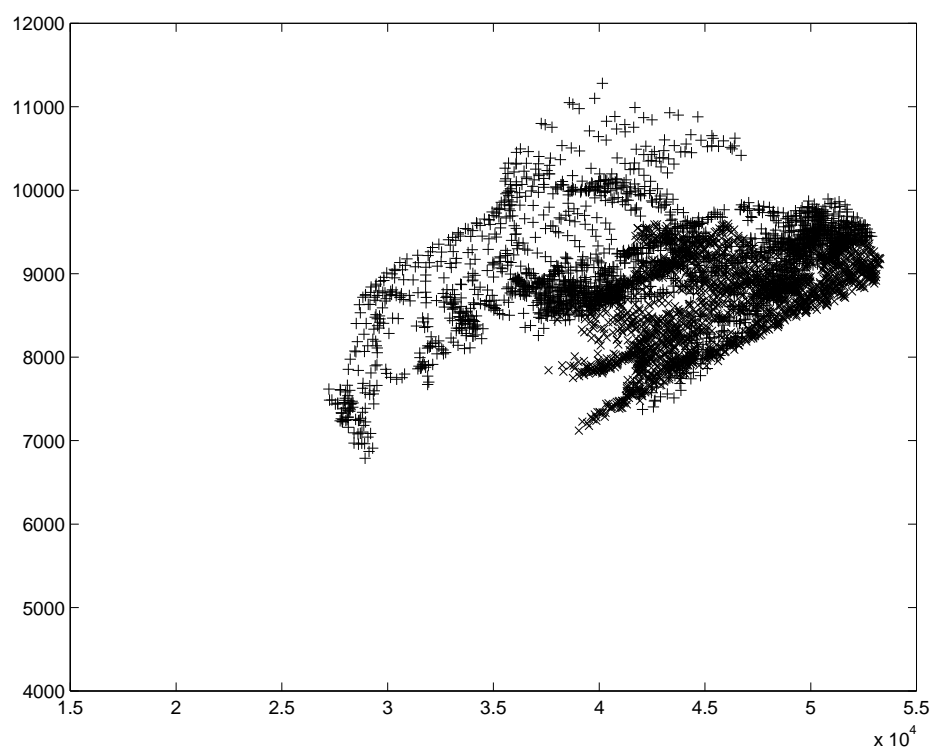

Figure 1: 2-class scatter-plot of test data with regard to two arbitrarily selected features 


\subsection{Experimental Setup}

Since we are primarily interested in testing the relative capabilities of the combination schemes, we shall consequently seek both to homogenize the classifiers constituting the combination, and to make them as representative of the pattern-data as possible. Thus, rather than the customary arrangement in which feature sets are allocated to a morphologically disparate set of classifiers on the basis of their individual representative strengths, we shall instead artificially impose a uniform classification scheme, a probability density function derived by regularly-spaced block-density histogramming of the pattern-data, upon each of the tested feature subsets constituting the combination.

Furthermore, in order that we might establish a direct measure of the classification performance of the various combination schemes, we shall impose the condition that the composite feature space PDF of $i$-dimensions which we are implicitly reconstructing by classifier combination is that obtained by a block density histogramming of the original $i$-dimensional space. In other words, we are designating the $i$ dimensional PDF thus derived as the underlying prior probability density function of the $i$-dimensional space.
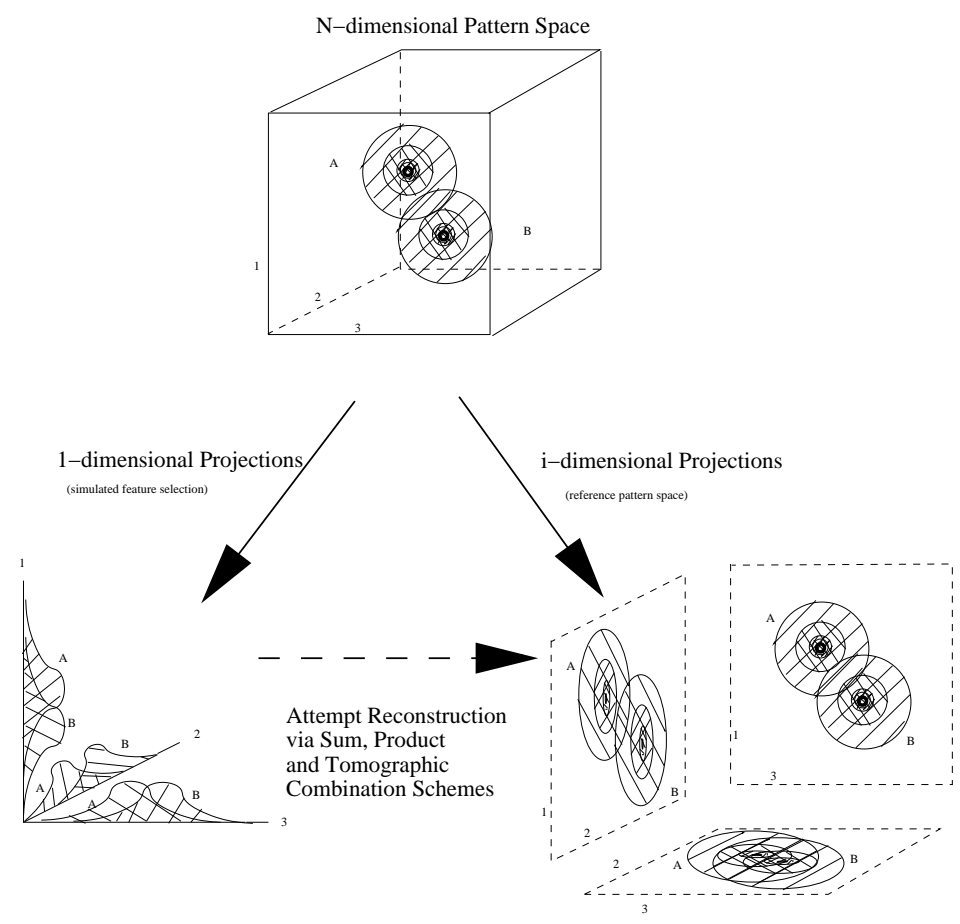

Figure 2: Experimental Format

For this approach to have general validity it is necessary that a large number of pattern vectors are sampled per histogram, even at the extremity of the tested dimensionality range. Thus we are also required to impose a relatively small number of bins per feature $(r)$ in order to maintain reasonable count statistics at the extremity of the range: of the order of $r=4$, given our $\approx 125000$ pattern vectors and 8 dimensional range.

Because of the need to establish a meaningful performance comparison across the dimensional range, it is additionally necessary to derive each of the tested multi-dimensional composite reference featurespace PDFs from the same experimental source. Hence we obtain the various $i$-dimensional spaces via projection of the complete $n$-dimensional pattern-space, finally averaging over all ${ }^{n} C_{i}$ performance figures thus obtained. Clearly, as the dimensionality $i$ varies, the averages thus obtained are subject to a statistical fluctuation associated with low number statistics (becoming asymptotic at $i=n$ when only one subspace exists), and hence the tested sequence is required to terminate well short of this 
value (coupled with the aforementioned consideration of avoiding under-sampling of the prior PDF at higher dimensionalities).

The reason it shall only prove necessary to consider the combination configuration consisting of $i$ one-dimensional classifiers (that is, combinations with one feature per classifier), is that we are principally interested in characterizing the variation of combination performance in relation to a uniform 'morphological information shortfall'. That is, we are primarily interested in the extent to which a combination scheme can make use of the $r i$ possible classifier ordinate values to reconstruct the $r^{i}$ possible $\underline{c o}$-ordinates of the prior PDF: introducing additional combinations of classifiers containing differing numbers of features would tend only to obscure this perspective without generating any additional insight into the combination processes not already encompassed by the latter approach. The experimental format for the real-world combination test is therefore as illustrated in Figure 2.

We should clarify that the test scenario in no way intended to represent a plausible real-world situation when feature-selection is explicitly taken into consideration: Given that we are in a position to obtain sufficient pattern-vectors as to be able to constrain a plausible model of the $i$-dimensional prior PDF, the most effective feature selection strategy (presuming a reasonably flexible set of classifier morphologies to choose from) would, most naturally, be to allocate the maximal $i$ features to the best performing classifier of the ensemble. Maximal retention of discrimination information is thereby guaranteed. We have, however, imposed the one-feature-per-classifier limitation in order that we might mimic the generalized situation in which any one-classifier parameterization of the whole $i$-dimensional space would likely be subject to serious over-parameterization error, and therefore disposed to reduce the classification rate in relation to a combination of classifiers of lower, but better sampled, feature dimensionalities. Of course, this condition being an external restriction means that, in fact, we do have access to a plausible model for the $i$-dimensional prior PDF as required for the purposes of performance evaluation.

The specified experimental scenario should thus be considered from the context of the broader tomographic perspective, within which feature-selection can be envisaged as seeking an appropriate balance between the mutually exclusive requirements: maximizing the retention of class-discriminant morphology information (through the allocation of spaces of higher feature dimensionalities to the classifiers), and the minimization of the dangers of over-classification (through the allocation of lower feature-space dimensionalities to classifiers).

The remaining aspect of the investigation, the assessment of the resilience to estimation error of the three fusion methods, is addressed in the above experimental context by the straightforward simulation of classifier error through adding uniform stochastic noise to each of the classifier density histograms (simulating, in effect, estimation-error arising from an insufficient degree of parameter-freedom amongst the classifiers, rather than estimation-error attributable to, say, incorrect, or over, parameterization). The tomographic performance results for the 'real-world' geological survey data are thus as depicted in Figure 6, of Section 4, alongside an analysis of its comparative significance. Placing the experiment in the widest context, however, requires that we turn, in the following section, to a more constrained model scenario.

\section{Investigation of the Comparative Tomographic Combina- tion Performance with Respect to Model Data}

The significance of the findings of the preceding investigation are, then, best established in relation to an absolute baseline against which the performance on real-world data may be graded. Any such proposed performance indicator must thus seek to determine the effect of classifier combination on the classification performance in a way that is independent of both pattern-data, as well as, classifier morphology. 
It so transpires that one of the very few classes of mathematically tractable characterizations of the algorithmic procedure of the appendix occurs in relation to prior PDFs composed of orthogonallygridded histograms of randomized density (hence fulfilling the requisite test conditions of independence to pattern-data morphology in the case where every such distribution is considered). Furthermore, it is natural to suppose that prior probability density distributions so derived will, when averaged over the ensemble, naturally constitute a generalized performance minimum for the tomographic methodology. This occurs as a consequence of its specifically seeking to reconstruct the overall pattern-space PDF through correlating morphology across the separate classifiers: a randomized morphology effectively undermines this agenda by decorrelating the differing subregions of the composite PDF, permitting the isolation of the required 'absolute' performance statistic. ${ }^{3}$

In combination with this argument, there is also the consideration that the randomization of the PDF morphology takes places with respect to a coordinatization aligned with the feature axes: tomographic methods, however, in applying a prior filtration to the back-projected radon data, implicitly seek to override metrics dictated by the feature axes in favor of those constrained solely by the underlying classifier morphology. There are therefore two distinct sets of reasons for supposing that the specified ensemble of prior PDF forms constitutes a generalized performance minimum with regard to the tomographic combination method, which (when combined with their unique mathematical tractability), naturally cement them as the choice for mathematical analysis of the relative tomographic combination performance. The derivation of this latter quantity will therefore occupy the majority of the remainder of the section.

An additional benefit arising from the elucidation of the model data performance statistics for all three of the tested combination methods is that, in doing so, we uncover a great many of the mathematical processes that underly the performance/dimensionality scaling phenomenon for classifier combiners in general. Remarkably, however, we shall demonstrate that the tomographic fusion method, notwithstanding the specified PDF restrictions, still exceeds the performance of the sum and product rules by a considerable margin.

\subsection{Tomographic Model Solution}

\subsubsection{General form of the composite tomographic PDF}

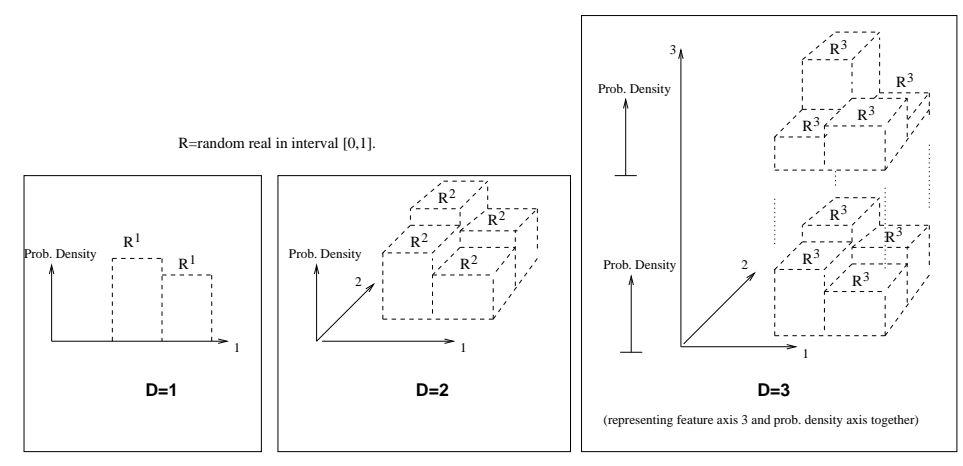

Figure 3: Randomized Composite PDF Morphology of Dimensionality $i=1,2,3$

The composite prior PDF format for the model solution is then as illustrated in Figure 3 for the first three dimensionalities in the range, with the obvious extrapolation to higher dimensionalities. The

\footnotetext{
${ }^{3} \mathrm{~A}$ performance maximum for the tomographic method is correspondingly established when the composite prior PDFs correspond to unimodal distributions that are capable of undergoing decomposition into intersecting hypercubes, in which case the tomographic combination performance achieves Bayes optimality (on the assumption of ideal constituent classifiers).
} 
per-ordinal resolution, $r$, of the gridded composite PDF having thus been lowered to a value of 2 , it becomes possible to uniquely grade the ordinal projections in the manner represented in Figure 4, the ordinal disparity thus, now, the single distinguishing parameter between classifier morphologies. That this reformatting is permissible within the context of the model data performance test is a consequence of the tomographic combination methodology's situation-specific independence to ordinate translations when the indicated prior PDF constraints are imposed, along with its more generalized independence to axial permutations occurring irrespective of any PDF model constraint. It shall therefore be the case, throughout the paper, that we continue the convention already adopted in Figure 4; namely, that calligraphic figures (such as $\mathcal{A}$ ) denote such magnitude-ordering of ordinal density values.

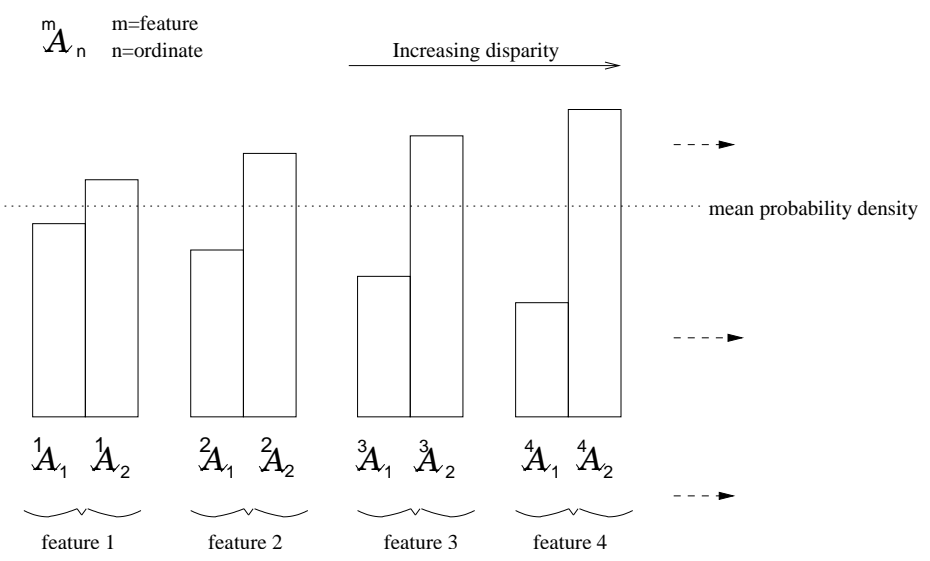

Figure 4: Projected Ordinal PDF Values for the Various 1-D Classifiers

The process outlined in appendix 1 may now be seen as a sequence of subtractions bringing the respective classifier ordinate values, ${ }^{x} \mathcal{A}_{2}$, into equality with the values of their neighboring ordinates, ${ }^{x-1} \mathcal{A}_{2}$, the number and magnitude of the subtractions enacted with respect to each set of $i$-dimensional ordinates thus dictating the tomographically estimated PDF value at the corresponding $\underline{c o-o r d i n a t e}$ constructed by their amalgamation. Since each subtraction removes a constant quantity, $\propto$ ( probability density $\times$ number of ordinates), from each $\mathrm{PDF}$, the corresponding per co-ordinate increment in the proposed composite PDF at each iteration increases in the geometric sequence $2^{1}, 2^{2}, 2^{3} \ldots$ Hence the actual value of the composite PDF proposed by tomographic fusion at a particular $i$-dimensional coordinate is thus:

$$
\left.P_{\text {tom. }}(\vec{a})={ }^{1} \mathcal{A}_{i}\left(\frac{1}{2}\right)^{i-1}+\sum_{m=1}^{\bar{a}}\left({ }^{2} \mathcal{A}_{i-m+1}-{ }^{1} \mathcal{A}_{i-m+1}-{ }^{2} \mathcal{A}_{i-m}+{ }^{1} \mathcal{A}_{i-m}\right)\left(\frac{1}{2}\right)^{i-m}\right)
$$

where $\vec{a}$ is the coordinate $\left(a_{1}, a_{2}, a_{3}, a_{4} . . a_{i}\right)$ ( $a_{x}$ may take the values 1 or 2 denoting the minimum and maximum ordinate values, respectively). The term $\bar{a}$ refers to the minimum $x$ value for which $a_{x}=1$. (We also specify that ${ }^{x} \mathcal{A}_{0}=0$ for consistency).

Thus we see that the predicted PDF value is governed by an accumulation of the disparities between ordinal projections, rather than simply those particular ordinates intersecting the point under consideration, as is the case for the sum rule and product rules (and indeed any other linear combination technique). In this way, even under the simplified scenario dealt with here, the tomographic technique involves all of the information contained within the ordinal projections (as the classifier PDFs) to generate the predicted prior probability density value. 


\subsubsection{Conditional form of the composite tomographic PDF}

The particular quantity that will be of interest to us in establishing the classification performance of the tomographic combination method is the PDF of the predicted composite probability density value at a particular $i$-dimensional coordinate in relation to a given composite probability value. That is, since we are seeking to establish a morphology-independent classifier combination performance estimate, we shall derive a predicted composite feature-space PDF value distribution function from the ensemble of all possible prior composite PDF morphologies within the terms set out above.

The first step in this process is to establish the ensemble average PDFs of the individual classifiers' projected ordinate values, $\left({ }^{x} A_{1,2}\right)$, in relation to a particular fixed prior PDF value, $X$, occurring at the coordinate $\left(a_{1}=1, a_{2}=1, a_{3}=1 \ldots a_{i}=1\right)$ (that is, fixed relative to the ensemble averaging over all prior composite PDFs consistent with this condition). Note that we are now in the original coordinate system, so the superscript numeral has no bearing on the relative value of $A$. The value of the prior density function at each $i$-dimensional coordinate (excluding $\left(a_{1}=1, a_{2}=1, a_{3}=1 \ldots a_{i}=1\right)$ ) is thus permitted to take, independently, a uniformly distributed random value in the interval $[0,1]$. (We need not consider the issue of normalization at this stage).

Once this quantity has been established, the resulting formulation will then permit a calculation of the disparity values, the predicted composite PDF value being constructed, as indicated, by a series of iterations whose total number is governed by the index number of first positive disparity value (of the $i$ total): that is, the first pair of feature ordinate values, ${ }^{x} A_{1,2}$, for which the unconstrained ordinate value ${ }^{x} A_{2}$ is greater than the constrained one; ${ }^{x} A_{1}$.

The degree to which the probability distribution of ordinal disparities is constrained by the actual value of the point under consideration depends, primarily, upon the dimensionality $i$ of the problem, a point we can elucidate by commencing with the calculation of the distribution of those ordinate projection values, ${ }^{x} A_{2}$, that do not intersect the point under consideration (and are therefore not in anyway constrained by it, given the randomness inherent in the PDF specification). This quantity is derived via convolution of the PDFs of the independent histogram density parameters comprising the composite feature-space PDF, ${ }^{x} A_{2}$ being essentially a sum over independent random variates:

$$
P\left({ }^{x} A_{2}\right) d^{x} A_{2}=\overbrace{\Pi \star \sqcap \star \ldots \sqcap}^{2^{i-1} \text { convolutions }} d A^{\prime} \equiv(\sqcap \star)^{2^{i-1}}
$$

(with the later term adopted as a convention throughout: $\sqcap$ is the probability density of the uniformly distributed random variate with limits $[0,1]$ )

That is, the distribution of ${ }^{x} A_{2}$ approaches the Gaussian form in the limit $i=\infty$ via the central limit theorem. Equation (2) may be written without explicit convolution as:

$$
P_{i}\left({ }^{x} A_{2}\right)=\frac{1}{2(i-1) !} \sum_{k=0}^{i}(-1)^{k}\left(\begin{array}{l}
i \\
k
\end{array}\right)\left({ }^{x} A_{2}-k\right)^{i-1} \operatorname{sgn}\left({ }^{x} A_{2}-k\right),
$$

via the characteristic function method.

Conversely, those ordinate projection values that do intersect the point under consideration (being therefore partially constrained by it), ${ }^{m} A_{1}$, are distributed thus:

$$
P\left({ }^{m} A_{1}-\vec{X}\right) d^{m} A_{1}=\overbrace{П \star \Pi \star \ldots \sqcap}^{2^{i-1}-1 \text { convolutions }} d^{m} A_{1} \equiv(\sqcap \star)^{2^{i-1}-1}
$$

where the constraining factor that the point $\left(a_{1}=1, a_{2}=1, a_{3}=1 \ldots a_{i}=1\right)$ must equate to the value $X$ acts to displace the distribution (minus one of the convolutions) by that same value (a point that may be readily confirmed by setting one of the $\Pi$ in equation (2) to a delta function centered on $X$ ). The probability that any given feature, $j$, has a disparity $D_{j}={ }^{j} A_{2}-{ }^{j} A_{1}$ between ordinate projections is hence: 


$$
P\left(D_{j} \mid X\right) d D_{j}=\int_{0}^{D}(\sqcap \star)^{2^{i-1}-1}\left({ }^{j} A_{1}-X\right)(\sqcap \star)^{2^{i-1}}\left({ }^{j} A_{1}+D_{j}\right) d\left({ }^{j} A_{1}\right) d D_{j}
$$

(with a negative value indicating ${ }^{j} A_{2}>^{j} A_{1}$ )

Recognizing the above as essentially a convolution with one of the functions having undergone the ordinate inversion $(A \rightarrow-A)$, giving a total of $2^{i-1}$ convolutions of the uniform distribution, we may re-write equation (5) as:

$$
P\left(D_{j} \mid X\right) d D_{j}=(\sqcap \star)^{2^{i-1}}\left(D-X-2^{i-1}\right) d D
$$

(the $-2^{i-1}$ term recentering the distribution to account for the [now implicit] ordinate inversion) Critically, whilst the individual sets of $A$ values for each of the ordinal projections are not independent of each other, their differentials $D$ are (perturbations of any particular $D$ value affect the ${ }^{x} A_{2}$ and ${ }^{x} A_{1}$ values of the other ordinates symmetrically). Hence, we can derive the probability distribution of the predicted tomographic combination rule $X$ values, $\left(P_{\text {tom. }}\right)$, by considering the various $D_{j}$ values independently, and making the appropriate $D$ for $A$ substitutions in equation (1). This comes about in the following way:

In the original coordinate system, the value $\bar{a}$ (the minimum $x$ value for which $a_{x}=1$ ) becomes instead $i$ minus the largest $x$ for which the corresponding $D_{x}$ value is less than zero (that is, for which ${ }^{1} A_{x}>^{2} A_{x}$ ), and the summation is hence over those $D$ magnitudes that are greater than $\left|D_{x}\right|$.

The revised format of equation (1) is therefore:

$$
P_{\text {tom. }}\left(a_{1}, a_{2}, \ldots a_{i}\right)=A_{\text {min }}\left(\frac{1}{2}\right)^{i-1}+\left|D_{i}\right|\left(\frac{1}{2}\right)^{i-1}-\left|D_{i-\bar{a}}\right|\left(\frac{1}{2}\right)^{i-\bar{a}}+\sum_{m=1}^{\bar{a}-1}\left|D_{i-m}\right|\left(\frac{1}{2}\right)^{i-m}
$$

(for $\bar{a}>2$, otherwise we would be required to remove the second and third terms as appropriate) Thus, for a given $\bar{a}$ the probability of a particular predicted prior PDF value, $P_{t o m}$. occurring at the point $(1,1, \ldots)$ with respect to an actual underlying value, $X$, is:

$$
\begin{array}{r}
P\left(P_{\text {tom. }} \mid \bar{a}, X\right)= \\
P\left(A_{\min } 2^{i-1}\right) \star P\left(\left|D_{i}\right| 2^{i-1}\right) \star P\left(-\left|D_{i-\bar{a}}\right| 2^{i-\bar{a}}\right) \star P\left(\left|D_{i-1}\right| 2^{i-1}\right) \star P\left(\left|D_{i-2}\right| 2^{i-2}\right) \star \ldots \star P\left(\left|D_{i-\bar{a}+1}\right| 2^{i-\bar{a}+1}\right)
\end{array}
$$

The probability that a particular $\mathrm{D}$ value is the most positive of the negative $D$ values (ie that $D=D_{\bar{a}}$ ) is given by:

$$
\begin{aligned}
P\left(D=D_{\bar{a}} \mid \bar{a}\right) & ={ }^{i} C_{1}\left[P\left(D>D_{\bar{a}} \& D<0\right)\right]\left[1-P\left(D>D_{\bar{a}} \& D<0\right)\right]^{i-1} \\
& ={ }^{i} C_{1}\left[\int_{0}^{D_{\bar{a}}} P(D) d D\right]\left[1-\int_{0}^{D_{\bar{a}}} P(D) d D\right]^{i-1}
\end{aligned}
$$

(the probability with which the number of terms, $\bar{a}$, is distributed being:)

$$
\begin{aligned}
P\left(\bar{a} \mid D_{\bar{a}}\right) & =\int_{0}^{-\infty}{ }^{i} C_{\bar{a}}\left[P\left(D<-D_{\bar{a}} \& D>0\right)\right]^{\bar{a}}\left[1-P\left(D<-D_{\bar{a}} \& D>0\right)\right]^{i-\bar{a}} P(D) d D \\
& =\int_{0}^{-\infty}{ }^{i} C_{\bar{a}}\left[\int_{0}^{-D_{\bar{a}}} P(D) d D\right]^{\bar{a}}\left[1-\int_{0}^{-D_{\bar{a}}} P(D) d D\right]^{i-\bar{a}} P(D) d D
\end{aligned}
$$

Given, then, that there are $\bar{a}$ terms, the probability distribution of those $D$ terms that do form the summation (that is the $D_{k}$ such that $1<k<\bar{a}$ ) is:

$$
P\left(D_{k} \mid D_{\bar{a}}, \bar{a}\right)={ }^{\bar{a}} C_{k}\left[P\left(D<D_{k}\right)\right]^{k-1} P\left(D=D_{k}\right)\left[P\left(D>D_{k} \& D<D_{\bar{a}}\right)\right]^{\bar{a}-1}[P(D>0)]^{i-\bar{a}}
$$


Thus, substituting the above in to equation (8), and eliminating $\bar{a}$ by summing over every possibility, we eventually obtain the sought quantity, $P_{t o m}$; the probability distribution of the predicted composite probability density value at $(1,1,1 \ldots)$ under tomographic combination with respect to the true value $X$ :

$$
P\left(P_{\text {tom. }} \mid X\right)=\sum_{\bar{a}=1}^{i} \prod_{k=1}^{k=\bar{a}} P\left(D_{k} \mid D_{\bar{a}}, \bar{a}\right) \int_{-\infty}^{-\infty} P\left(\bar{a} \mid D_{\bar{a}}\right) P\left(D_{\bar{a}}\right) d D_{\bar{a}} P\left(P_{\text {tom. }} \mid \bar{a}, X\right)
$$

(the outstanding term, $A_{\text {min }}$, in the above being eliminated by summing over the two possibilities $A_{\min }={ }^{x} A_{1}, A_{\min }={ }^{x} A_{2}$ ). For our purposes, it will be sufficient to carry out this integration numerically.

\subsubsection{Classification Rate}

Given that the tomographic method makes optimal use of the morphological information contained in the classifiers constituting the combination, the variance of this distribution then gives us some indication of the absolute loss of composite PDF descriptivity that occurs following feature-selection with respect to increasing dimensionality (since we have averaged over a full set of randomized morphologies). However, it is the loss of classification information with which we are most concerned.

The average misclassification rate with respect to full gamut of $i$ dimensional PDF morphologies under the tomographic scheme is, then, given as the integral:

$$
\begin{gathered}
\int_{X_{1}=X_{2}}^{X_{1}=\infty} \int_{X_{2}=0}^{X_{2}=\infty} \int_{P_{\text {tom. }}^{1}=P_{\text {tom. }}^{2}}^{P_{\text {tom. }}^{1}=\infty} \int_{P_{\text {tom. }}^{2}=0}^{P_{\text {tom. }}^{2}=\infty} X_{2} \cdot P\left(P_{\text {tom. }}^{1} \mid X_{1}\right) \cdot P\left(P_{\text {tom. }}^{2} \mid X_{2}\right) d P_{\text {tom. }}^{1} d P_{\text {tom. }}^{2} d X_{1} d X_{2} \\
+\int_{X_{2}=X_{1}}^{X_{2}=\infty} \int_{X_{1}=0}^{X_{1}=\infty} \int_{P_{\text {tom. }}^{2}=P_{\text {tom. }}^{1} P_{\text {tom. }}^{2}=\infty}^{\int_{P_{\text {tom. }}=0}^{1}=X_{10 m .}=\infty} X_{1} \cdot P\left(P_{\text {tom. }}^{1} \mid X_{1}\right) \cdot P\left(P_{\text {tom. }}^{2} \mid X_{2}\right) d P_{\text {tom. }}^{1} d P_{\text {tom. }}^{2} d X_{1} d X_{2}
\end{gathered}
$$

where the sub/superscripts 1 and 2 indicate class labels.

That is, we sum over the two sets of possibilities for which errors occur: $\left\{P_{\text {tom. }}^{1}>P_{\text {tom. }}^{2}\right.$. when $\left.X_{2}>X_{1}\right\}$ and $\left\{P_{\text {tom. }}^{2}>P_{\text {tom. }}^{1}\right.$ when $\left.X_{1}>X_{2}\right\}$. A numerically computed graph of the outcome of this equation for a range of dimensionalities is given in Figure 5.

\subsection{Sum Rule Model Solution}

Turning now to the equivalent formulation for the sum rule combination scheme, the predicted value of the composite feature-space probability density at point $(1,1,1 \ldots)$ for a randomized morphology, $\left(P_{\text {sum }} \mid X\right)$, is given, for an underlying value $X$, by the formula:

$$
P_{\text {sum }} \mid X=\sum_{m=0}^{m=i}{ }^{m} A_{1} /\left(i 2^{i-1}\right)
$$

(we here introduce a normalization $\left(i 2^{i-1}\right)$ for consistency with the stochastic approach above).

The calculation of the way in which this quantity is distributed is complicated by the fact that many of the terms implicit in the individual summation, ${ }^{x} A_{1}$, are also implicit in a number of the other summations (specifically, at the various intersections of the hyper-planes represented by the ${ }^{x} A_{1}$ ). However, by explicitly acknowledging that each of the constituent hyper-planes essentially constitutes a sum over all of the points of the composite posterior PDF having coordinates with consecutive ordinates held at unity, we can isolate the various independent coordinate values in multiples: 


$$
P\left(P_{\text {sum }} \mid \vec{X}\right)=\sum_{k=0}^{k=i}\left[k \sum_{m=1}^{m=i C_{k}} X_{m}\right]
$$

(where the $X_{m}$ are independently selected from the distribution $\sqcap$ ).

The summation over every $X_{m}$ for a particular $k$ thus represents the set of $k$ coordinates having equal numbers of ordinals of value 1 .

When the ensemble average is sought over every possible randomized morphological permutation of the composite prior pattern-space PDF in the previous manner, the predicted prior PDF value at $(1,1,1 \ldots)$ is thus distributed as:

$$
P(H \mid \vec{X})=\left(1(\sqcap \star)^{i} C_{1}\right) \star\left(2(\sqcap \star)^{i} C_{2}\right) \star\left(3(\sqcap \star)^{i} C_{3}\right) \star \ldots\left(i(\sqcap \star)^{i} C_{i}\right.
$$

The calculation of the misclassification rate is then achieved as before (equation 15), via an integration over every probability for which the predicted value of class 1 at $(1,1,1 \ldots), P_{\text {sum }}^{1}$, is of the opposing magnitude to the equivalent point of class $2, P_{\text {sum }}^{2}$, in relation to the actual value disparity: again see Figure 5 for a numerical calculation of this quantity against dimensionality.

\subsection{Product Rule Model Solution}

The calculation of misclassification rate with respect to randomized morphology for the product rule is considerably more involved in the previous cases as a consequence of the proliferation of terms with mixed products of higher variate powers as dimensionality increases. As such, the misclassification rate verses dimensionality calculation may, very possibly, not be generally formalizable except on a case-bycase basis. A partial mathematical treatment may, however, be encompassed by approximation: that is, by explicitly assuming the independence of the summed terms in each ordinal projection from their counterparts in the remaining ordinal projections.

The probability density distribution of each ordinal is then as derived previously:

$$
P\left({ }^{x} A_{2}\right) d^{x} A_{2}=(\sqcap \star)^{2^{i-1}}
$$

However, it is the multiplicative value density of these terms with which we are primarily interested:

$$
P\left(P_{\text {prod. }} \mid X\right)=P\left({ }^{1} A_{1} *^{2} A_{1} * \ldots *^{z} A_{1} \mid X\right)
$$

(overlooking normalization considerations).

Hence, we need to apply a logarithmic substitution in order to render the distribution tractable as a convolution of random variates:

$$
\begin{gathered}
P\left(\log \left(P_{\text {prod. }}\right)\right)=P\left(\log \left({ }^{1} A_{1}\right)+\log \left({ }^{2} A_{1}\right)+\ldots \log \left({ }^{z} A_{1}\right)\right) \\
\left.\Rightarrow P\left(\log \left(P_{\text {prod. }}\right)\right)=P\left(\log \left({ }^{1} A_{1}\right)\right) \star P\left(\log \left({ }^{2} A_{1}\right)\right) \star \ldots P\left(\log \left({ }^{z} A_{1}\right)\right)\right)
\end{gathered}
$$

The distribution resulting from this substitution is thus approximately log-normal (increasingly so as dimensionality increases via the multiplicative central limit theorem): Montecarlo simulation for the lower end of the dimensional range tends to confirm the accuracy of the adopted approximation.

The performance results in terms of the misclassification rate for the product rule are as given in Figure 5 via the formulation of equation 15, which, along with the previous results, thus serves as our baseline performance, 'noise response' model over the dimensional range: direct comparison with the results for the 'real-world' geological survey data given in Figure 6 are thus invited. We re-emphasize in passing that the vertical ordinate of graph 6 represents the ensemble average error-rate: the error bars thus 
refer to the unbiased estimate of the standard deviation of the mean of the error rate (a figure which would otherwise be partially dictated by the number of samples, equal to ${ }^{26} C_{i}$, contributing to each test point, $i$, of the dimensional range). This consideration, however, does not supersede the fact of an inevitably incremental correlation amongst the individual samples as the dimensionality increases (in consequence of a greater degree of overlapping amongst the feature subsets), manifesting itself as a decreasing sample variance with increasing $i$ (without, in principle, affecting the mean to any great degree). Hence we opt to terminate the sequence at a figure significantly smaller than the total dimensionality to mitigate the impact of this effect.

We also note that in the wider interpretation of Figures 5 and 6 , the horizontal graph axis could be equivalently labeled 'classifier number' rather then 'composite feature space dimensionality', the results being intended to be at least indicative of the more general fusion scenario for which classifiers are not necessarily limited to representing single features (via the argument of Section 3).

\section{Analysis of Results}

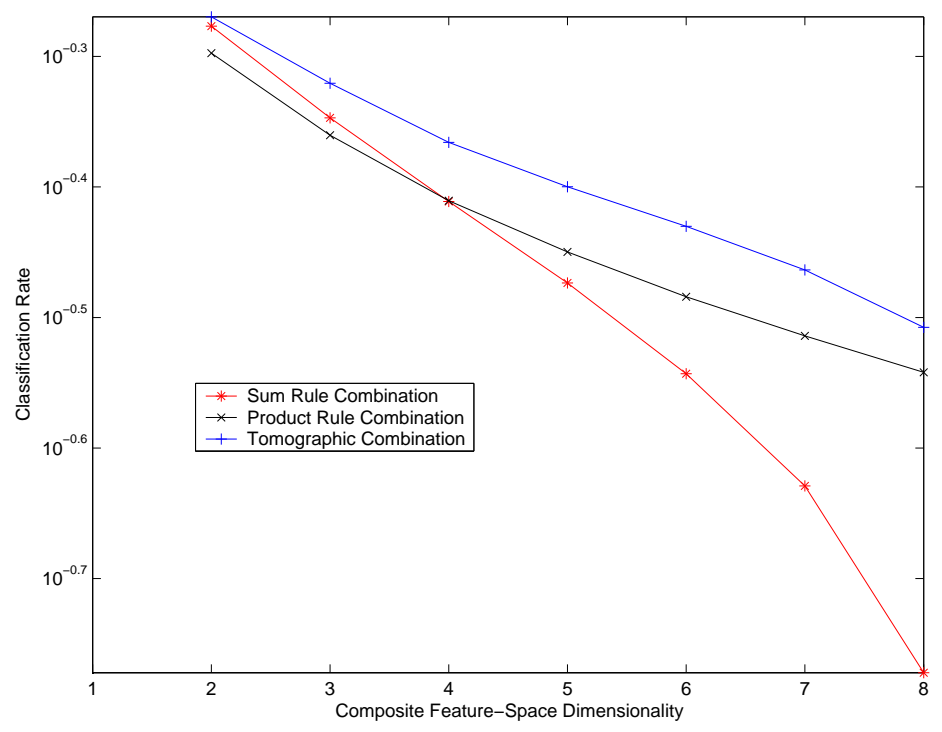

Figure 5: Classification Rate vs Dimensionality for the Model Data

In presenting an analysis of the results quantified in Figures 5 and 6, the first point to notice (briefly alluded to earlier) is that, even though the tomographic method is disadvantaged by the specifically randomized nature of the morphology in the 'base-line' performance test ${ }^{4}$, the performance graph of Figure 5 suggests that it is, in terms of classification performance, nonetheless the superior combination methodology at every point of the dimensional range with respect to the sum and product rule alternatives. That is, the tomographic method, by virtue of making use of all of the data available in the classifiers constituting the combination (utilizing the cross-referenced information contained within every classifier ordinal, rather than just those constituting the implicitly reconstructed $c o$-ordinate), is able to recover a greater extent of the composite pattern-space's PDF lost during the feature selection process than can existing methods (as represented by the sum and product rules via the argument of $[17])$.

The differences between the reconstructive abilities of the methods are encapsulated in the distributions $P\left(P_{\text {tom. }} \mid X\right), P\left(P_{\text {sum }} \mid X\right)$ and $P\left(P_{\text {prod. }} \mid X\right)$, describing the deviation from the prior composite probability

\footnotetext{
${ }^{4}$ Hence imposing an absolute minimum of correlatable morphology between the various classifiers' PDFs.
} 


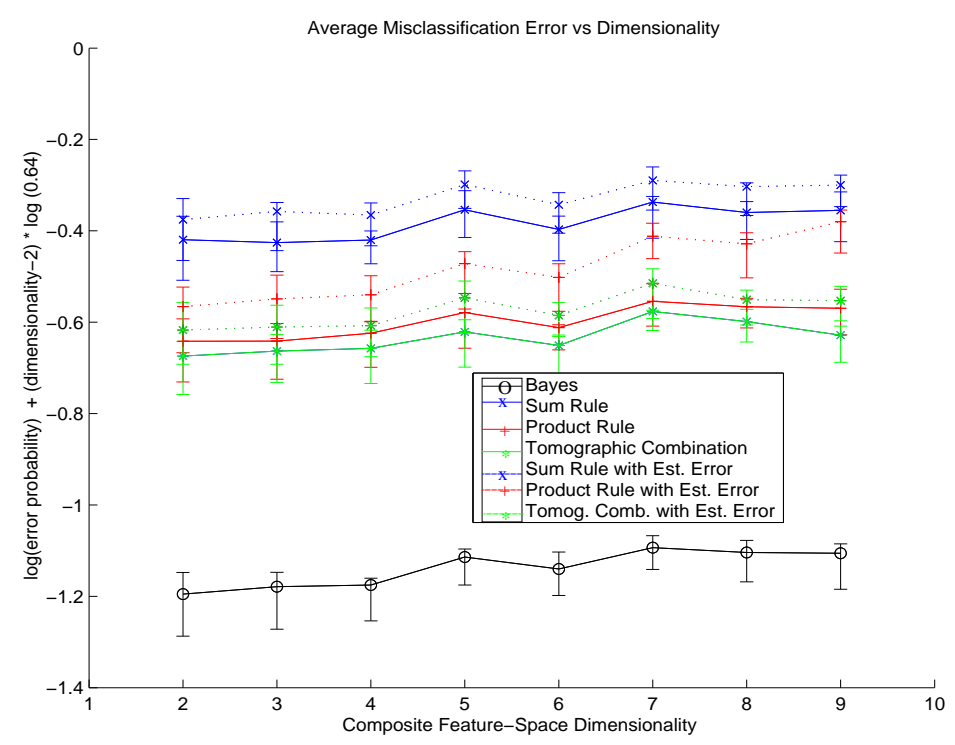

Figure 6: Misclassification Rate vs Dimensionality for the Real-World Data (note linearizion of vertical scale)

density value at the implicitly reconstructed pattern-space co-ordinate. We observe that the performance results of the three methods are not especially different relative to the simulated Bayes error rate (which descends logarithmically). This we hence speculate to be a consequence of the fact that the variances of these distributions are of similar orders of magnitude for constant dimensionalities, being governed chiefly by the number of self-convolutions of the uniform function, with higher numbers thus progressively approximating the Gaussian form (or log-Gaussian form, in the case of the product rule). This 'convolution number' being the same for the sum, product and tomographic combination methods means that the difference between the techniques, the 'methodological signature', as it were, is manifested solely by the differences in the shape of the distribution functions.

Thus, in retrospect, we can appreciate that any combination method postulating a composite PDF solution consistent with the ordinal projection values (as the sum, product and tomographic methods all do), will prove to give very similar classification accuracies when the class morphologies are randomized over all ensembles. It is, as stated earlier, only when correlated classifier morphologies can be related to each other that advantages of tomographic combination come significantly to light.

Turning then to the real-world performance tests, for which we postulated that correlated morphology amongst the classifiers is the rule rather that the exception, the equivalent results for the rock-strata data (Figure 6) would seem to indicate that the ability of the tomographic combiner to correlate morphology between the $i$ discrete classifiers is much more in evidence, with a clear performance advantage over the sum and product rules developing with increasing dimensionality.

In terms of the point-by-point relationship between the three combining methodologies, it would appear that the tomographic method more closely mimics the performance of the product rule than the sum rule, despite its origins in the latter technique. We hypothesize that this is a consequence of actual independence in the original PDFs being recovered by the tomographic method (which is feasible, given that, on inspection, the prior PDFs have an approximately similar morphology to the Gaussian distribution of uniform covariance). It should be noted, however, that the tomographic estimationerror graph more closely parallels that of the sum rule than the product rule (as we would conceivably expect, given the results of 'base-line' performance measure tests of Figure 5). Thus, in a sense, Figure 5 and its attendant mathematical derivations can be considered to additionally serve as an indicator of the isolated effects of estimation error on the respective tomographic, sum and product combination 
rules (the estimation error plots in Figure 6 then correspondingly being seen as contextual indicators of the effects of estimation error). That is, the point-by-point randomness of the prior PDFs in the model solution gives rise to a noise function at each of the classifier ordinates (the sum of the independent random variables being binomially distributed) that behaves similarly to the simulated estimation error of the second investigation, albeit without the context of the real-world classifier PDFs.

\section{Conclusions}

\subsection{Summary of Results}

In terms of the advocation of a general combination strategy for an unfamiliar classification problem on the basis of the tests we have conducted, it would seem that the tomographic method is the indicated approach, both in terms of its reconstructive ability, as well as in its estimation error resilience (for which the method mimics the performance of the sum rule, without that technique's reconstructive deficiencies). In particular, these advantages would appear to scale favorably with the number of classifiers constituting the combination.

It must, however, be clearly understood that the scatter of data points in Figure 6 is such that it is not possible to guarantee in all cases (or even much more than half of the cases for the lower dimensionalities) that the tomographic method is optimal (it being always possible to consider composite pattern-space PDF morphologies that favor either of the alternative strategies). Our argument, we emphasize, is with respect to arbitrary underlying PDF morphologies ${ }^{5}$, for which the presence of backprojection artifacts implied by conventional linear combination methods (the gamut of which the sum and product rules are deemed to collectively encompass) are taken to be generally unrepresentative. It is interesting to note, however, on the evidence of Figure 6, that in the real world scenario, despite the presumed presence of these artifacts, the product rule would appear to be significantly better at composite PDF morphology recovery than the sum rule. This is presumably a consequence of the fact that the reconstruction artifacts are suppressed (but, note, not fully removed) via repeated multiplication. This advantage, however, is generally suppressed by the multiplicative cumulation of estimation-error effects for all but ideal classifiers.

\subsection{Outlook}

With respect to the prospects for further improving the tomographic combination methodology, one possibility is to note that the modified Högbom method specified in the appendix inherently regards the rectanguloid hypercube as its deconvolution primitive, and thus constitutes only a partial realization of the potential for applying tomographic filtration to combined classifiers (the central idea of which is removal of all axial bias from back-projected radon data). Clearly, while the rectanguloid hypercube primitive serves to remove much of the the feature axial alignment imposed by classifier combination (in particular, the elongated striations depicted in Figure 2 of [1]), it still exhibits an obvious axial alignment on the local scale. Thus there is scope for future methodological improvement by introducing more rotationally symmetric primitives (for instance hyper-ovoids, which would be capable of reconstructing complete Gaussians).

Another, complementary, approach is to seek to increase the computational performance of the tomographic method, which at present, though considerably economized [4] (and parametrically tunable to a high degree), falls significantly behind the linear sum and product methods. To remedy this situation, it is necessary to employ a pre-filtration approach. That is, we should have to apply a filtering convolution to the individual classifier PDFs and combine via the sum rule, imposing a positivity condition

\footnotetext{
${ }^{5}$ as distinct from the randomized morphologies of our model solution.
} 
on the output. Such a method, while conjectured to be of somewhat less accuracy than the current approach, would have the benefit of scaling linearly in terms of operation time with the number of classifiers. To determine exactly what the accuracy deficit might be for such a procedure would be the basis of further empirical and theoretical investigation.

It might also be of interest to consider alongside an investigation of this type the suitability of differing base classifiers as candidates for pre-filtration. In principle our method is completely independent with regard to underlying base classifier type (as argued in [1] on a theoretical level, an also experimentally in relation to NN, k-NN and Gaussian classifiers in various combinations). However, the practical pay-off in each case might not be justified; efficient pre-filtration prior to tomographic reconstruction requires that there exists a mathematical representation of the base classifier PDFs amenable to convolution (such as could, for instance, be determined even for non-Bayesian classifiers like decision trees by the explicit elaboration of their recursive division of the feature space into hyperplane sections). If such a direct formulation is not straightforwardly available, it would be necessary to construct it artificially via sampling and interpolation; in itself a form of PDF estimation, and thus prone to an additional source of estimation error. In some cases one may therefore be justified in compromising accuracy in the interests of reducing computation time. Equally, one may prefer handling combination in a manner conceptually congruent with the underlying base classifier paradigm (for instance, employing a neural net combination layer for combining neural nets). However, while morphologically consistent, such approaches (unlike tomographic combination) can never be considered morphologically unbiased.

In sum, then, we have provided performance statistics to complement the earlier theoretical assertion that tomographic combination recovers the greatest degree of the composite pattern space PDF morphology lost during feature selection (the precise quantity of recoverable information being indicated by the disparity between the Bayesian and tomographic error rates for Figures 5 and 6 with regard to artificial and real situations, respectively).

Moreover, we have demonstrated that the tomographic method, as well as having the best underlying performance rate, has also a similar error resilience to the sum-rule combination methodology. We have thereby combined the best of both of the aspects of combination through which classification performance is improved, the morphologically reconstructive and the error-negating: these two aspects being previously partially, though separately, represented within the product and sum rules, respectively.

\section{Acknowledgment}

This research was carried out at the University of Surrey, UK, supported by, and within the framework of, EPSRC research grant number GR/M61320.

\section{Appendix 1: Step-by-Step Approach to Procedurally Im- plementing Post-filtered Tomographic Classifier Combina- tion}

The following is a line-by-line guide to procedural implementation of Högbom (post-filtered) tomographic deconvolution. In the interests of clarity, it is immediately followed by an equivalent, though marginally more computationally intensive description in terms of functional maps.

1. Assemble experts constituting the combination as a series of PDFs ranging over $n$ discrete feature spaces of respective dimensionality; $a_{1}, a_{2} \ldots a_{n}$ for the class set; $\omega_{1}, \omega_{2}, \ldots \omega_{m}$; label them $P_{n}\left(\vec{X}_{n}\right)$, where $\vec{X}_{n}$ ranges over the vector space of dimensionality $a_{n}$. 
2. Select the first class of the series, $\omega_{1}$, and establish peak probability density value(s), $P_{n}^{\max }$, for of each expert's individual representation of that class.

3. Specify a pair of accuracy parameters, $\Delta z$ and $\Delta x$, that respectively denote the probability density and feature-space resolutions.

4. Establish the 'hyper-area' between the probability density ordinates representing the peak value and (peak value $-\Delta z$ ) for each of the classifier PDFs: ie, the scalar number of $(\Delta x)^{a_{i}} \times \Delta z$ units between the two probability density values for each of the classifiers in the fusion. Vectors within these bounds are designated $\vec{X}_{n}^{\prime}$.

5. Specify a matrix of dimension; $a_{1}+a_{2}+\ldots+a_{n}$ with each element designating an (initially zero) probability density value attributable to every $(\Delta x)^{a_{1}+a_{2}+\ldots+a_{n}}$ unit of the composite featurespace. ${ }^{6}$ Add a value, $N$, to those points representing all combinations of $n$ concatenations of the respective (co-)ordinates established in 4: That is, the Cartesian product $\left\{\vec{X}_{1}^{\prime}\right\} \times\left\{\vec{X}_{2}^{\prime}\right\} \times\left\{\vec{X}_{3}^{\prime}\right\} \times$ $\ldots \times\left\{\vec{X}_{n}^{\prime}\right\} .\left(N\right.$ must be $\left.>\sum_{i=1}^{n} P_{n}^{\max }\right)$.

6. Subtract the resolution parameter $\Delta z$ from each peak value $P_{n}^{\max } ; \forall i$, and set an iteration parameter (say, $t$ ) to zero.

7. Subtract a quantity $\left|X_{1}^{\prime}\right| \times\left|X_{2}^{\prime}\right| \ldots \times\left|X_{i-1}^{\prime}\right| \times\left|X_{i+1}^{\prime}\right| \times \ldots \times\left|X_{n}^{\prime}\right| \times d z$ from the current peak value of each classifier, $P_{n}^{\max } ;\left|X_{j}^{\prime}\right|$ being the scalar values derived in $\mathbf{5}$, ie: the number of coordinate vectors $\left\{\vec{X}_{i}^{\prime}\right\}$ of dimensionality $a_{i}$ counted by the PDF hyper-area establishing procedure above. Note, especially, the absence of $\left|X_{i}^{\prime}\right|$ in the product entity.

8. Establish the new hyper-area value associated with the subtraction 7, that is: the hyper-area between the probability density ordinates representing the previous and current peak-values (as per 4).

9. Allocate a value $N-t . \Delta z$ to those points in the deconvolution matrix representing novel coordinates established after the manner of $\mathbf{4}$. That is, the Cartesian product difference:

$\left[\left(\left\{\vec{X}_{1}^{\prime}\right\}_{\text {old }} \cup\left\{\vec{X}_{1}^{\prime}\right\}_{\text {new }}\right) \times\left(\left\{\vec{X}_{2}^{\prime}\right\}_{\text {old }} \cup\left\{\vec{X}_{2}^{\prime}\right\}_{\text {new }}\right) \times \ldots \times\left(\left\{\vec{X}_{n}^{\prime}\right\}_{\text {old }} \cup\left\{\vec{X}_{n}^{\prime}\right\}_{\text {new }}\right)\right]-\left[\left\{\vec{X}_{1}^{\prime}\right\}_{\text {old }} \times\left\{\vec{X}_{2}^{\prime}\right\}_{\text {old }} \ldots\left\{\vec{X}_{n}^{\prime}\right\}_{\text {old }}\right]$

( $t$ the cycle count number, $N$ as above).

10. Increment the cycle counter, $t$, by 1 and go to 7 while $P_{n}^{\max }>0, \forall i$.

11. After termination of the major cycle $\mathbf{7 - 1 1}$, subtract a value $t . \Delta z$ from each point of the deconvolution matrices to establish true PDFs, if required (see footnote 5).

12. Repeat from 2 for the remaining classes in the sequence $\omega_{1}, \omega_{2} \ldots \omega_{m}$.

As a functional mapping we thus seek to repeatedly perform the conditional iteration:

\footnotetext{
${ }^{6}$ In a memory-restricted environment, it is alternatively possible to perform iterations 7-11 simultaneously for the respective classes, retaining only those points of coincidence between the various class probabilities: a significantly smaller set than the matrix specified in 5. The total memory footprint for this configuration is of the order $\{X\}^{a_{1}}+\{X\}^{a_{2}}+$ $\ldots\{X\}^{a_{n}}$, rather than the former; $\{X\}^{a_{1}+a_{2}+\ldots+a_{n}}$ (for feature-spaces of uniform dimensional size $X$ ); which is to say, an equivalent memory requirement to conventional linear methods of combination.
} 


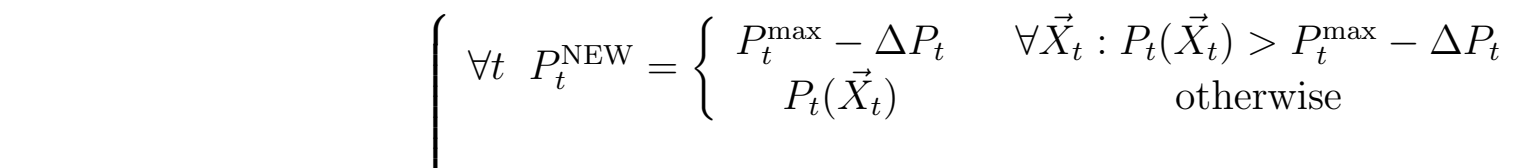

$$
\begin{aligned}
& \text { where } \Delta P_{t}=\Delta z \times\left|\vec{X}_{1}: P_{1}\left(\vec{X}_{1}\right)=P_{1}^{\max }\right| \\
& \times\left|\vec{X}_{2}: P_{2}\left(\vec{X}_{2}\right)=P_{2}^{\max }\right| \times \ldots \times\left|\vec{X}_{t-1}: P_{t-1}\left(\vec{X}_{t-1}\right)=P_{t-1}^{\max }\right| \\
& \times\left|\vec{X}_{t+1}: P_{t+1}\left(\vec{X}_{t+1}\right)=P_{t+1}^{\max }\right| \times \ldots \times\left|\vec{X}_{n}: P_{n}\left(\vec{X}_{n}\right)=P_{n}^{\max }\right| \\
& \text { while } \left.\exists \vec{X}_{t}: P_{t}\left(\vec{X}_{t}\right)>0\right\} \\
& P_{\text {TOM }}^{\mathrm{NEW}}\left(\vec{X}_{\mathrm{TOM}}\right)=\left\{\begin{array}{cc}
P_{\mathrm{TOM}}\left(\vec{X}_{\mathrm{TOM}}\right)+\Delta z & \forall \vec{X}_{\mathrm{TOM}}, t: P_{t}\left(\vec{X}_{\mathrm{TOM}} \cdot \vec{X}_{t}\right)> \\
P_{t}\left(\vec{X}_{\mathrm{TOM}}\right) & P_{t}^{\max }-\Delta P_{t} \\
\text { otherwise }
\end{array}\right. \\
& \forall \vec{X}_{\mathrm{TOM}} \quad P_{\mathrm{TOM}}\left(\vec{X}_{\mathrm{TOM}}\right)=P_{\mathrm{TOM}}^{\mathrm{NEW}}\left(\vec{X}_{\mathrm{TOM}}\right) \\
& \forall \vec{X}_{t}, t \quad P_{t}\left(\vec{X}_{t}\right)=P_{t}^{\mathrm{NEW}}\left(\vec{X}_{t}\right)
\end{aligned}
$$

The final $P_{\text {TOM }}$ function is hence our tomographically reconstructed probability density function. (Note that it is initiated with uniform zero values across the vectorial range; ie $\left.P_{\mathrm{TOM}}\left(\vec{X}_{\mathrm{TOM}}\right)=0 \forall \vec{X}_{\mathrm{TOM}}\right)$.

\section{References}

[1] D. Windridge, J. Kittler, “A Morphologically Optimal Strategy for Classifier Combination: Multiple Expert Fusion as a Tomographic Process", IEEE PAMI, Vol 25, no. 3, March 2003

[2] D. Windridge, J. Kittler, "Classifier Combination as a Tomographic Process", Multiple Classifier Systems, LNCS. Vol. $2096,2001$.

[3] D. Windridge, J. Kittler, "A Generalized Solution to the Problem of Multiple Expert Fusion", Univ. of Surrey Technical Report: VSSP-TR-5/2000)

[4] D. Windridge, J. Kittler, "An Efficient Algorithmic Implementation of Tomographic Classifier Combination", Journal of the Pattern Recognition Society, Submitted

[5] D. Windridge, J. Kittler, "Morphologically Unbiased Classifier Combination Through Graphical PDF Correlation", LNCS 2396, August 2002

[6] D. Windridge, J. Kittler, "On the General Application of the Tomographic Classifier Fusion Methodology, LNCS. Vol. 2364, June 2002

[7] Windridge D., Kittler J., "Combined Classifier Optimization via Feature Selection", Proceedings "Advances in Pattern Recognition", Lecture Notes in Computer Science. vol. 1876

[8] K. M. Ali and M.J. Pazzani, "On the link between error correlation and error reduction in decision tree ensembles", Technical Report 95-38, ICS-UCI, 1995

[9] T.K. Ho, J.J. Hull, and S.N. Srihari, "Decision combination in multiple classifier systems", IEEE Transactions on Pattern Analysis and Machine Intelligence, vol. 16, no. 1, 1994, 66-75.

[10] L. Xu, A. Krzyzak and C.Y. Suen, "Methods of combining multiple classifiers and their application to handwriting recognition", IEEE Trans. SMC, vol. 22, no. 11, 1994, 1539-1549 
[11] Intrator N. and Cohen S., "Automatic model selection in a hybrid perceptron/radial network", Information Fusion 3 (2002); 259-266

[12] Breiman L., Bagging predictors, Machine Learning 1996; vol. 24, no. 2:123-140.

[13] Drucker H., Cortes C., Jackel L. D., Lecun Y., and Vapnik V., Boosting and other ensemble methods, Neural Computation, 1994; vol. 6, no. 6:1289-1301

[14] Bruzzone L., Cossu R. and Vernazza G., "Combining parametric and non-parametric algorithms for a partially unsupervised classification of multitemporal remote-sensing images", Information Fusion 3 (2002); 289-297

[15] Dietterich T. G., Bakiri G. "Solving Multiclass Learning Problems via Error-Correcting Output Codes." Journal of Artificial Intelligence Research, 1995; Vol 2.: 263-286.

[16] R A Jacobs, "Methods for combining experts' probability assessments", Neural Computation, 3, pp 79-87, 1991

[17] J. Kittler, M. Hatef, R.P.W. Duin, and J. Matas, "On combining classifiers", IEEE Transactions on Pattern Analysis and Machine Intelligence, vol. 20, no. 3, 1998, 226-239

[18] L. Lam and C.Y. Suen, "Optimal combinations of pattern classifiers", Pattern Recognition Letters, vol. 16, no. 9, 1995, 945-954.

[19] A F R Rahman and M C Fairhurst, "An evaluation of multi-expert configurations for the recognition of handwritten numerals", Pattern Recognition Letters, 31, pp 1255-1273, 1998

[20] A F R Rahman and M C Fairhurst, "A new hybrid approach in combining multiple experts to recognise handwritten numerals", Pattern Recognition Letters, 18, pp 781-790, 1997

[21] K Woods, W P Kegelmeyer and K Bowyer, "Combination of multiple classifiers using local accuracy estimates", IEEE Trans. Pattern Analysis and Machine Intelligence, 19, pp 405-410, 1997

[22] J. Kittler, "Improving Recognition Rates by Classifier Combination: A Review", 1st IAPR TC1 Workshop on Statistical Techniques in Pattern Recognition, 205-210, June 1997 